\title{
Characteristics of Large-Vessel Occlusion Associated with COVID-19 and Ischemic Stroke
}

\author{
(D) J. John, (DP. Kesav, (D) V.A. Mifsud, (D). Piechowski-Jozwiak, (D). Dibu, (D)A. Bayrlee, (D) H. Elkambergy, (D)F. Roser,
} (D) M.S. Elhammady, (DK. Zahra, and (IDS.I. Hussain

\begin{abstract}
SUMMARY: The mechanisms and phenotype of ischemic stroke associated with coronavirus disease 2019 (COVID-19) remain uncertain. A retrospective study was conducted in patients with COVID-19 presenting with ischemic stroke from March 1 to May 25, 2020, and cases with large-vessel occlusion were identified. To provide baseline institutional stroke data within and outside the COVID-19 pandemic, all consecutive ischemic stroke and TIA admissions (COVID and non-COVID) to the hospital during a 10-week period from March 1 to May 10, 2020, were collected and compared with data from the same time period in 2019. Among 20 patients with COVID-19 and acute ischemic stroke, 15 (75\%) had large-vessel occlusion. These patients were young (mean age, 46.5 years), male (93\%), without major burden of traditional cardiovascular risk factors, and had a severe stroke presentation. Largevessel occlusions were observed in multiple vessels (40\%), uncommonly affected vessels, and atypical locations with a large thrombus burden. Systemic thrombosis separate from large-vessel occlusion was not uncommon (26\%). At short-term follow-up, stroke etiology remained undetermined in $46 \%$ of patients and functional outcome was poor. The above findings raise the possibility of stroke related to mechanisms induced by the COVID-19 infection itself, including a hypercoagulable state and/or endothelial damage. In addition, they document the severe presentation and poor outcomes of large-vessel occlusion in COVID-19 ischemic stroke.
\end{abstract}

ABBREVIATIONS: CCA = common carotid artery; COVID-19 = coronavirus disease 2019; LVO = large-vessel occlusion; SARS CoV-2 = Severe Acute Respiratory Syndrome coronavirus-2

C oronavirus disease 2019 (COVID-19) is an ongoing pandemic caused by infection with the Severe Acute Respiratory Syndrome coronavirus-2 (SARS CoV-2). ${ }^{1,2}$ There are now multiple reports of COVID-19 affecting the central nervous system, ranging from meningitis/encephalitis to stroke. ${ }^{3-5}$ In a single-center study of 214 hospitalized patients with COVID-19 from Wuhan, China, where the infection first occurred, up to $36.4 \%$ of patients had neurologic manifestation, including acute cerebrovascular disease with severe and nonsevere infection in $5.7 \%$ and $0.8 \%$ of these patients, respectively. ${ }^{3}$ In addition, there are also reports of ischemic stroke being caused by large-vessel occlusion (LVO) in patients with COVID-19 without significant pre-

Received June 15, 2020; accepted after revision July 24.

From the Department of Neurology (S.J., P.K., V.A.M., B.P.-J., S.I.H.), Neurological Institute; Neurointerventional Surgery (S.J., M.S.E., K.Z., S.I.H.), Neurological Institute; Neurocritical Care Unit (J.D., A.B., H.E.), Critical Care Institute; and Department of Neurosurgery, Neurological Institute (F.R., M.S.E.), Cleveland Clinic, Abu Dhabi, United Arab Emirates.

Please address correspondence to Seby John, MD, Neurointerventional Surgery, Neurological Institute, C-07-231, Cleveland Clinic Abu Dhabi, Al Maryah Island, Abu Dhabi, UAE; e-mail: johns5@clevelandclinicabudhabi.ae

- Indicates open access to non-subscribers at www.ajnr.org

Indicates article with supplemental on-line table.

http://dx.doi.org/10.3174/ajnr.A6799 existing cardiovascular risk factors. ${ }^{6}$ While the reasons for ischemic stroke in COVID-19 are unclear, hypotheses of an inflammatory cytokine storm-triggered hypercoagulable state, endothelial damage, and arrythmias have been postulated. ${ }^{7,8}$ However, as it stands, the mechanisms, phenotype, and optimal management of ischemic stroke associated with COVID-19 still remain uncertain.

There is an urgent need to identify associations and predictors of severity, morbidity, and mortality in patients with ischemic stroke and COVID-19, especially in the LVO subgroup, given that it is most disabling.

\section{MATERIALS AND METHODS}

This is a single-center, retrospective, observational study. All consecutive patients who were admitted to the hospital with a diagnosis of COVID-19 from March 1 to May 25, 2020, were identified. These patients tested positive on SARS CoV-2 polymerase chain reaction testing via nasopharyngeal and oropharyngeal swabs or on sputum samples collected when intubated. Among the above cohort, all patients with ischemic stroke and LVO were identified.

To provide baseline institutional stroke data within and outside the COVID-19 pandemic, we collected all consecutive ischemic stroke and TIA admissions (COVID and non-COVID) to 
the hospital during a 10-week period from March 1 to May 10, 2020, and compared then with the data from the same time period in 2019.

Retrospective data collection points included details regarding demographics, stroke risk factors, clinical presentation, stroke scales, imaging results and laboratory investigations, acute treatments including intravenous thrombolysis and endovascular thrombectomy, time metrics, stroke classification and etiology, ischemic stroke subtype classification based on the Trial of Org 10172 in Acute Stroke Treatment (TOAST), ${ }^{9}$ clinical outcomes, and discharge disposition. Regarding the COVID-19 infection, additional information was collected including non-neurologic COVID-19 symptoms, chest imaging, and treatment details specific for COVID-19.

Institutional review board approval was obtained before pursuing this study.

\section{Statistical Methods}

For baseline data, means and SDs were calculated for continuous variables, while categoric variables were expressed as counts and percentages. $P$ values associated with comparisons on continuous variables, categoric variables, and count variables were calculated using independent-samples $t$ tests, Fisher exact tests, and $\chi^{2}$ tests, respectively. All statistical analyses were performed using Microsoft R Open 3.5.1 software (https://mran.microsoft.com/). The significance threshold was set at a 2 -sided $P$ value $<.05$.

\section{Findings}

When we compared admissions during the 10-week period from March 1 to May 10 between 2019 and 2020, there was a significant increase in the number of ischemic strokes in 2020 (76 versus 103, $P=.044$ ), while TIA remained unchanged (33 versus $27, P=.439$ ). LVO, including occlusion of the ICA, M1 and M2 segments of the MCA, and the basilar artery, was significantly higher in 2020 (20 [18.3\%] versus 44 [33.8\%], $P=.008)$. When we compared time metrics between 2019 and 2020, presentation to the hospital from a mean last-known-well time (620.6 \pm 743.7 versus $516.6 \pm$ 556.86 minutes, $P=.293$ ) and mean door-to-needle times for intravenous thrombolysis $(35.5 \pm 12.7$ versus $42.7 \pm 14.8$ minutes, $P=.171)$ were similar in both years. However, door-to-groin puncture times for endovascular thrombectomy were significantly longer in 2020 (67.75 versus 104.30 minutes, $P=.001$ ).

From March 1 to May 25, six hundred seventy-three patients with COVID-19 were admitted to the hospital. Among these, 20 (2.97\%) patients presented with acute ischemic stroke. Of these patients, 15 (75\%) had documented LVO.

The On-line Table details the characteristics of patients with COVID-19 with ischemic stroke and LVO. The mean age at presentation was 46.5 years (range, 23-66 years), with 11 (73.3\%) patients being 50 years of age or younger. This cohort was overwhelmingly male $(14,93.3 \%)$. Nine $(60 \%)$ patients did not have any traditional cardiovascular risk factors for stroke. In the remaining patients, hypertension was present in 3 (20\%); hyperlipidemia, in 1 (6.67\%); diabetes mellitus, in 4 (26.67\%); and coronary artery disease, in 1 (6.67\%) patient. The maximum number of concurrent risk factors was 2, which $3(20 \%)$ patients possessed. With regard to COVID-19 symptoms, 6 (40\%) had fever, 5 (33.3\%) had cough or shortness of breath, and 6 (40\%) were asymptomatic. Headache before stroke was present in $3(20 \%)$ patients. Nine $(60 \%)$ patients had pneumonia on chest x-ray or chest CT performed at or shortly after admission. Average C-reactive protein levels (closest to the time of stroke) and D-dimer levels (highest level) were $106.2 \mathrm{mg} / \mathrm{L}$ (range, $0.4-328.3 \mathrm{mg} / \mathrm{L}$ ) and $2.34 \mathrm{mcg} / \mathrm{mL}$ fibrinogen equivalent units (range, $1.24-4 \mathrm{mcg} / \mathrm{mL}$ ), respectively.

The mean NIHSS score at presentation was 21.5 (range, 038). A single patient with vertebral artery V4 segment occlusion with cerebellar strokes and gait ataxia had NIHSS $=0$, but most patients had severe deficits at presentation. Twelve (80\%) patients had anterior circulation stroke. On CTA of the head and neck, 7 patients (46.7\%) had isolated occlusion of the MCA M1 segment. Two (13.3\%) patients had tandem occlusion of the ICA and MCA, 2 (13.3\%) patients had tandem occlusion of the common carotid artery (CCA), ICA, and MCA; and 1 (6.7\%) patient had an occlusion in the M1 MCA but with concurrent subclavian artery thrombosis. Of the $3(20 \%)$ patients with posterior circulation stroke, 1 (6.7\%) had occlusion of the basilar artery and a separate posterior cerebral artery P2 segment occlusion. The other $2(13.3 \%)$ patients had vertebral artery occlusion in the V4 segment and throughout its course soon after the origin, respectively. In total, multiple vessel occlusions were present in 6 (40\%) patients, with large thrombus burden in these cases. Of note, there were $4(26.7 \%)$ patients with other systemic thrombosis, including pulmonary embolus and vein thrombosis.

Figure 1 demonstrates patient 13 who had a large subocclusive thrombus at the ICA origin and tandem MCA occlusion. This patient did not have any underlying atherosclerotic disease in the ICA as demonstrated by postthrombectomy images, which showed a normal carotid bifurcation. Figure $2 A,-B$ demonstrates patient 2 with discrete thrombus in the proximal ICA without underlying atherosclerotic disease followed by complete occlusion distally and tandem MCA occlusion. Figure 2 shows a large subocclusive thrombus in the CCA and complete occlusion of the proximal CCA, with a large thrombus burden in patients 6 (Fig $2 C, D$, and $E$ ) and 11 (Fig $2 F,-G$ ), respectively, with more distal complete occlusion of the ICA and MCA in both cases. Finally, Patient 12 (Fig $2 H,-I$ ) had a large subocclusive thrombus in the proximal subclavian artery and MCA occlusion. All cases demonstrated in Fig 2 did not have obvious features of underlying atherosclerotic disease. Figure 3 details patterns of infarcts with multiple infarcts in different vascular distributions.

Treatment with intravenous thrombolysis was administered in $3(20 \%)$ patients, and 6 (40\%) underwent mechanical thrombectomy, including 5 patients with isolated M1 occlusion and 1 with tandem cervical ICA and M1 occlusions. Successful recanalization of TICI score $2 \mathrm{~b}-3$ was achieved in 4 (66.7\%), with a mean groin-to-recanalization time of 28.75 minutes (range, 17-53 minutes). In cases of successful recanalization, aspiration alone was used in three-quarters of cases, while combined stent retriever and aspiration were used in a single case. The 2 cases without recanalization were MCA occlusion from atherosclerotic disease. One patient underwent multiple thrombectomy and balloon angioplasty attempts without success, and the other had vessel rupture after a single pass of aspiration, which was the only complication in all thrombectomy cases. At discharge or 30 days 


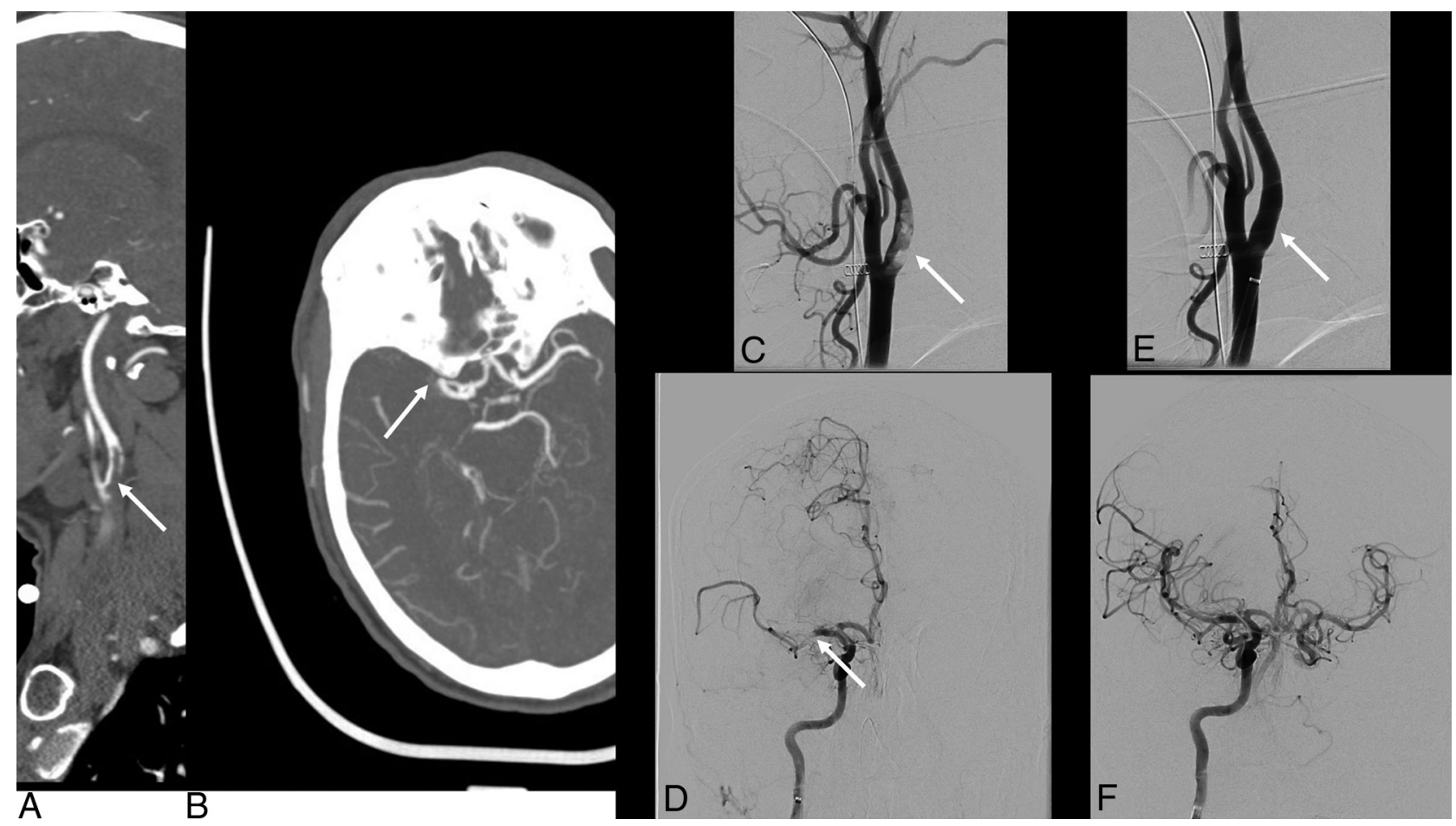

FIG 1. Patient 13 . CTA of the head and neck reveals a large subocclusive clot at the right carotid bifurcation extending into the proximal ICA ( $A$, arrow) and tandem right M1 MCA occlusion ( $B$, arrow). The same was confirmed on DSA when the patient was taken for mechanical thrombectomy ( $C$ and $D$, arrow). Postthrombectomy DSA shows complete resolution of extracranial carotid thrombus with no underlying atherosclerotic disease (E, arrow) and recanalization of the MCA occlusion $(F)$.

poststroke, 1 patient achieved an mRS score of $0-2$, one achieved an mRS score of 3 , and none died.

For the whole cohort, 7 (46.7\%) patients had stroke of undetermined etiology; 4 (26.7\%), from intracranial atherosclerotic disease; and 3 (20\%), from a cardioembolic source. Only 3 (20\%) patients achieved a good outcome of mRS 0-2 at discharge or 30 days poststroke, and 1 (6.7\%) patient died in the hospital. Mortality was secondary to severe cerebral edema secondary to cardiac arrest.

\section{DISCUSSION}

This retrospective study on COVID-19 and ischemic stroke has revealed multiple findings. There is a high rate of LVO in patients with ischemic stroke and COVID-19. In this series, $75 \%$ of patients with COVID-19 with ischemic stroke had LVO. Even if excluding the single patient with vertebral artery occlusion from possible dissection, the rate remains at $70 \%$, which is considerably higher than that usually observed from historical controls. These patients were overwhelmingly male and young without a significant burden of traditional cardiovascular risk factors and had severe stroke presentation with or without systemic features related to COVID-19.

Traditionally the United Arab Emirates has had a younger age of onset for stroke with male predominance as a result of poorly controlled vascular risk factors among expatriate migrant male workers. ${ }^{10,11}$ The mean age of patients in the 10-week study period presented above for 2019 and 2020 was approximately 58 years $(33.4 \%$ of patients overall were 50 years of age or younger) with up to $70 \%$ being male. Our comparison with historical controls confirms an even younger age of onset in patients with COVID-19 with ischemic stroke.

Unique patterns of LVO were observed in the patients with COVID-19 with ischemic stroke. Up to $40 \%$ of patients had $>1$ vessel with thrombosis or occlusion. In the anterior circulation, large thrombus burden was seen in uncommonly affected vessels and in atypical locations. These observations, along with the finding of separate systemic thrombosis in one-quarter of patients, suggest an association between a COVID-19-mediated hypercoagulable state and thromboembolism. ${ }^{12-15}$ Further studies looking at inflammatory and hypercoagulable markers will need to be performed to establish the pathophysiology of COVID-19 in ischemic stroke.

There were 4 patients with LVO secondary to presumed intracranial atherosclerotic disease, including 3 with severe presentation from MCA occlusion. It is interesting that all 3 patients had pneumonia on chest imaging. Two of the 3 patients with M1 MCA occlusion secondary to intracranial atherosclerotic disease had only a deep basal ganglia infarct, despite having a large mismatch on perfusion imaging and nonrecanalization of the vessel. It is plausible that factors such as hypoxia or hypotension associated with COVID-19 pneumonia with or without acute respiratory distress syndrome may have resulted in acute decompensation of cerebrovascular autoregulation, which was previously preserved in the setting of chronic vascular occlusion or severe stenosis (Fig 4). Correction of hypoxia or hypotension may have prevented a larger territory infarct, assuming that collaterals were previously well- 


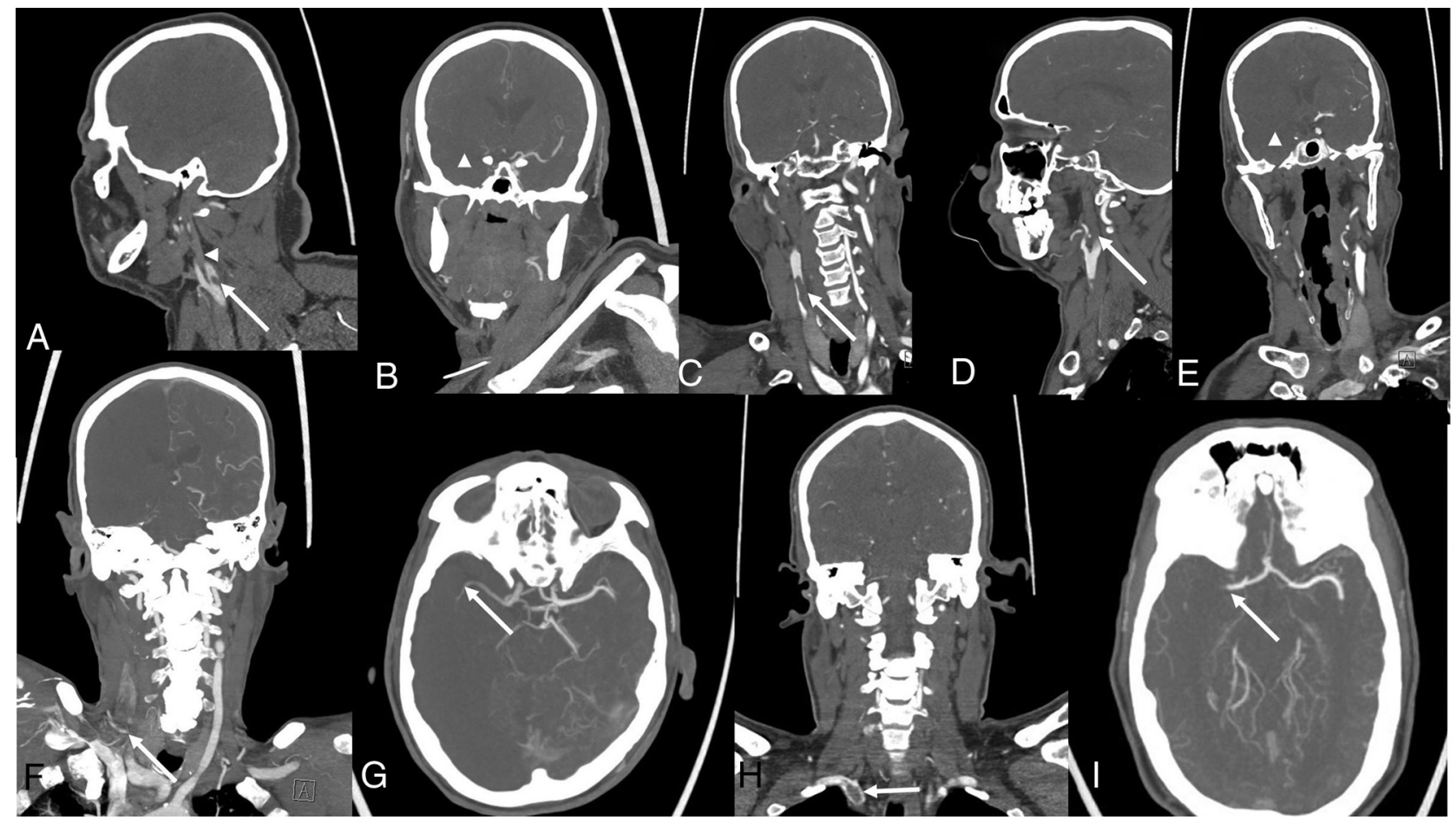

FIG 2. Patient 2. CTA of the head and neck reveals discrete thrombus in the proximal ICA without underling atherosclerotic disease (A, arrow) followed by complete occlusion distally in the ICA ( $A$, arrowhead) and tandem MCA occlusion ( $B$, arrowhead). Patient 6 . CTA reveals a large subocclusive thrombus in the right CCA (C, arrow) followed by complete occlusion of the ICA ( $D$, arrow) and MCA (E, arrowhead). Patient 11. CTA reveals complete occlusion of proximal right CCA with a large thrombus burden $(F$, arrow) followed by complete occlusion of the right ICA and tandem M2 MCA occlusion (G, arrow). Patient 12. CTA reveals a large subocclusive thrombus in the proximal right subclavian artery (H, arrow) and tandem right M1 MCA occlusion (l, arrow).

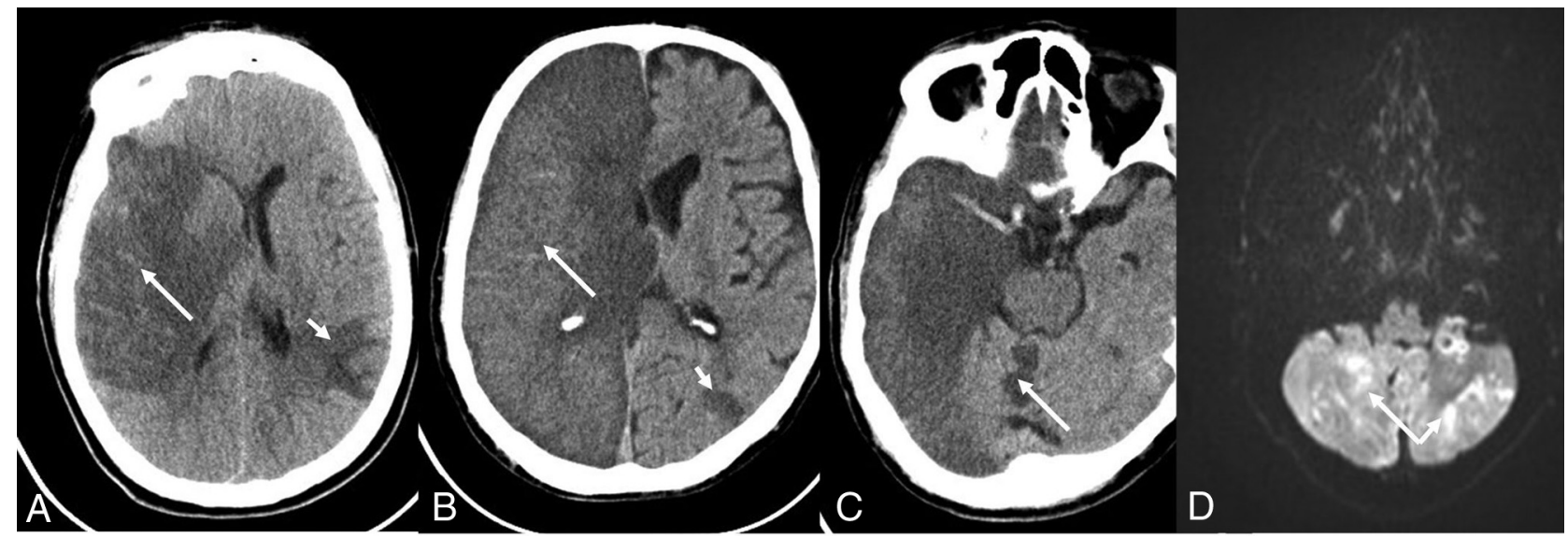

FIG 3. Patterns of infarcts. Patient 9 . CT of the head shows a large right MCA-distribution infarct (A, long arrow) secondary to right M1 MCA occlusion, but it also shows a smaller infarct in the left parietal lobe ( $A$, short arrow). Patient 11. CT of the head shows a complete right hemispheric infarct $(B$, long arrow) but also small left parietal ( $B$, short arrow) and right cerebellar $(C$, arrow) infarcts. Patient 14 . Diffusion-weighted sequence on MR imaging shows scattered areas of infarcts in bilateral cerebellar hemispheres ( $D$, arrows).

developed in these patients. Also, complete occlusion of underlying stenotic lesions causing LVO may have been an acute phenomenon due to in situ thrombosis secondary to a virus-induced hypercoagulable state, but this cannot be confirmed.

Despite only short-term follow-up data being available, close to one-half of patients had stroke of undetermined etiology. In the background of the cohort involving predominantly young patients, without significant burden of cardiovascular risk factors, this finding again raises the possibility of stroke related to mechanisms induced by the COVID-19 infection itself. Although the cohort is small and only short-term follow-up is available, functional outcomes in these patients were poor, with only a minority of patients achieving a good outcome of mRS $0-2$. This result could be related to multiple factors, the foremost of which is 


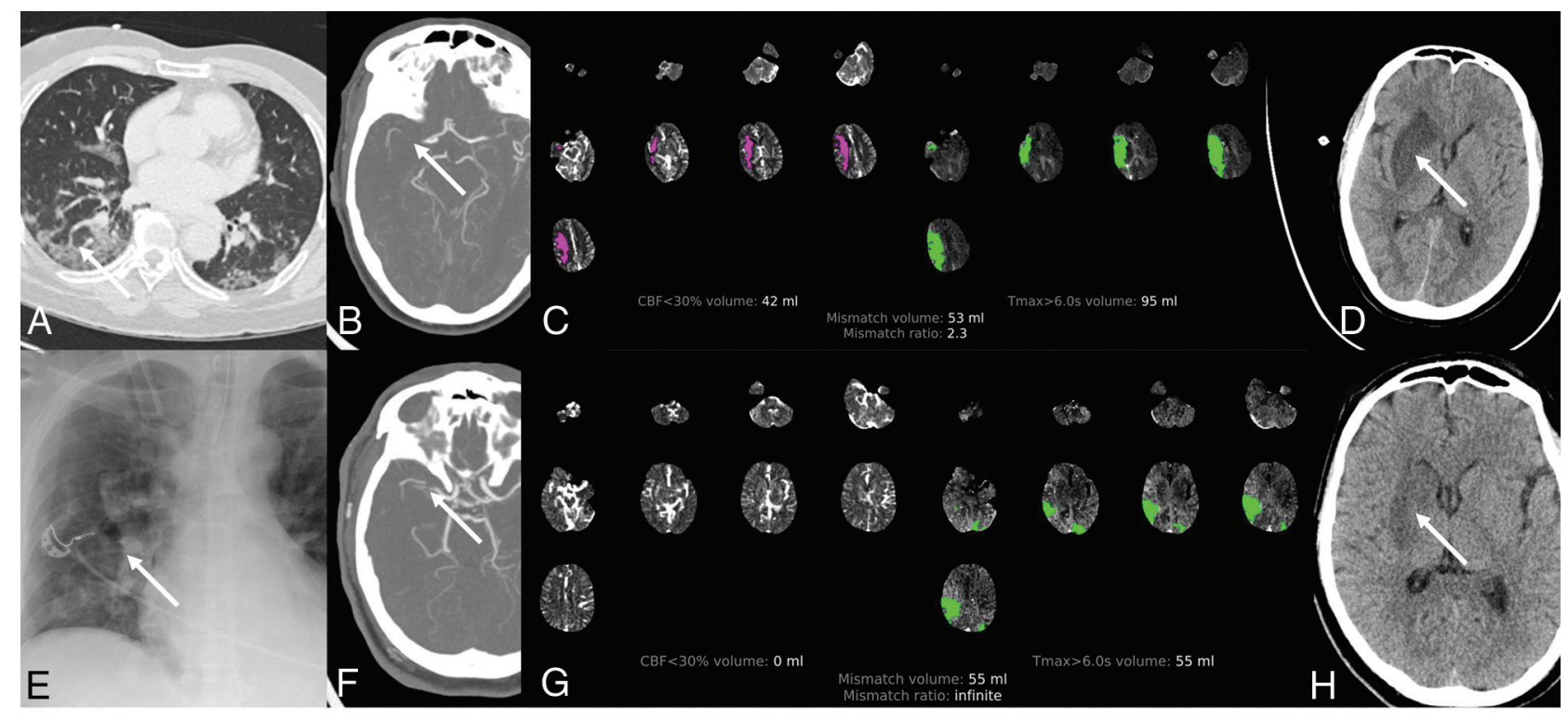

FIG 4. Patient 4 . CT of the chest demonstrates diffuse lung parenchymal changes associated with viral pneumonia ( $A$, arrow). CTA of the head shows right M1 MCA occlusion ( $B$, arrow), and the CT perfusion maps show a large area of penumbral mismatch (C). However, follow-up CT of the head shows a smaller right basal ganglia final infarct $(D$, arrow). Patient 8 . Chest $x$-ray shows multifocal infiltrates (E, arrow). CTA of the head shows right M1 MCA occlusion or critical stenosis ( $F$, arrow), and CT perfusion maps show a large area of penumbral mismatch (G). Follow-up CT of the head shows a smaller right basal ganglia final infarct $(H$, arrow) despite nonrecanalization following endovascular thrombectomy.

because many patients were ineligible for thrombectomy at the time of presentation due to a large burden of established infarct or severe systemic disease. Historically, LVO subtype of stroke is the most disabling. The added insult of systemic disease from COVID-19 causing pneumonia and acute respiratory distress syndrome with consequent cerebral oxygenation and hemodynamic alterations may, in part, explain the poor outcomes. Patients with severe COVID-19 who require intubation and prolonged intensive care unit stays are prone to complications, including hypotension and cerebral hypoperfusion, stress cardiomyopathy and resultant decreased left ventricular ejection fraction, and atrial fibrillation with or without a rapid ventricular response. In addition, severe COVID-19 has been associated with a cytokine storm and hyperviscosity. ${ }^{16}$ Progression to disseminated intravascular coagulation is more common in COVID-19, with 1 report documenting an incidence of $8.7 \%$ with associated $94 \%$ mortality. ${ }^{17}$ Even in those patients who underwent thrombectomy and achieved successful recanalization, an mRS of $0-2$ in $25 \%$ of patients was the best outcome achieved at short-term follow-up.

Our results are largely consistent with a few published small series of COVID-19 and ischemic stroke ${ }^{6,18,19}$ with regard to younger age of onset along with less prevalence of traditional stroke risk factors among patients with COVID-19 with stroke. Oxley et $\mathrm{al}^{6}$ published a case series of 5 young patients with COVID-19 and LVO, 3 (60\%) of whom had vascular risk factors for stroke. In the case series of 32 patients in Yaghi et al, ${ }^{18} 11$ were 50 years of age or younger, of whom $45 \%$ had vascular risk factors. However, it is not clear how many of these patients had LVOs.

While there is the clear limitation of a small sample size and lack of follow-up, the above results serve at least to raise awareness of the severe presentation and outcomes of LVO in COVID-
19 ischemic stroke. Compared with many reports on drastic decreases in stroke admission, our institution recorded an increase in stroke admissions. This could be explained by other centers no longer taking care of such patients during the current pandemic and by a possible alteration in referral patterns because our institution continued to operate as a center of excellence for cerebrovascular care in addition to managing patients with COVID-19. In addition, the increase in the volume of patients with stroke cannot be generalized to imply an increase in the incidence of stroke during the pandemic. The increase in LVO in 2020 may possibly be related to the high incidence of LVO in COVID-19, but this again cannot be conclusively confirmed. With regard to our institutional stroke pathway workflow, there was no significant increase in door-to-needle times for intravenous thrombolysis for ischemic stroke during the pandemic. However, a significant delay in door-to-groin times for mechanical thrombectomy was observed. This can be explained by the institution of a protected code stroke in our institution based on recommendations by various societies and reflects the impact of the pandemic as a real-world experience. ${ }^{20}$

The study has limitations including its retrospective design, single-center setting, and small sample size. There could have been a referral bias of only more severe patients with COVID-19 and stroke being referred to our institution. However, this scenario is unlikely because larger facilities that became dedicated COVID-19 hospitals were mandated to transfer all patients with stroke, and smaller hospitals continued to transfer patients with stroke as per established transfer protocols similar to pre-COVID times. Alternatively, patients with stroke and COVID-19 and LVO could have been admitted to other centers during the study period. Only short-term follow-up of patients is available at the time of this writing. 


\section{CONCLUSIONS}

There is a high incidence of LVO in patients with COVID-19 presenting with ischemic stroke. These patients are young men, without significant burden of traditional cardiovascular risk factors and have severe stroke presentation with or without systemic features related to COVID-19. LVOs were observed in multiple vessels, uncommonly affected vessels, and atypical locations with a large thrombus burden. Systemic thrombosis separate from LVO was not uncommon. The etiology of the stroke remained undetermined in up to one-half of patients. The above findings raise the possibility of stroke related to mechanisms induced by the COVID-19 infection itself, including an induced hypercoagulable state and/or endothelial damage. Functional outcome was poor in this cohort at short-term follow-up.

\section{REFERENCES}

1. Huang C, Wang Y, Li X, et al. Clinical features of patients infected with 2019 novel coronavirus in Wuhan, China. Lancet 2020;395:497506 CrossRef Medline

2. Guan WJ, Ni ZY, Hu Y, et al; China Medical Treatment Expert Group for Covid-19. Clinical characteristics of coronavirus disease 2019 in China. N Engl J Med 2020;382:1708-20 CrossRef Medline

3. Mao L, Jin H, Wang M, et al. Neurologic manifestations of hospitalized patients with coronavirus disease 2019 in Wuhan, China. JAMA Neurol 2020;77:683 CrossRef Medline

4. Zhou Y, Li W, Wang D, et al. Clinical time course of COVID-19, its neurological manifestation and some thoughts on its management. Stroke Vasc Neurol 2020;5:177-79 CrossRef Medline

5. González-Pinto T, Luna-Rodríguez A, Moreno-Estébanez A, et al. Emergency room neurology in times of COVID-19: malignant ischemic stroke and SARS-COV2 infection. Eur J Neurol 2020 April 30. [Epub ahead of print] CrossRef Medline

6. Oxley TJ, Mocco J, Majidi S, et al. Large-vessel stroke as a presenting feature of Covid-19 in the young. N Engl J Med 2020;382:e60 CrossRef Medline

7. Qin $\mathrm{C}$, Zhou L, Hu Z, et al. Dysregulation of immune response in patients with COVID-19 in Wuhan, China. Clin Infect Dis 2020;71:762-68 CrossRef Medline
8. Zhang Y, Xiao M, Zhang S, et al. Coagulopathy and antiphospholipid antibodies in patients with Covid-19. N Engl J Med 2020;382: e38 CrossRef Medline

9. Adams HP Jr, Bendixen BH, Kappelle LJ, et al. Classification of subtype of acute ischemic stroke: definitions for use in a multicenter clinical trial-TOAST: Trial of Org 10172 in Acute Stroke Treatment. Stroke 1993;24:35-41 CrossRef Medline

10. Jozwiak BP, Kumar V, Hussain S, et al. Cleveland Clinic Abu Dhabi stroke registry (CCADSR) young ischemic strokes: initial results. J Neurol Sciences 2019;405:85 CrossRef

11. Khan M, Hashim H, Nisa Z, et al. Thrombolysis for acute ischemic stroke: experience in Dubai, and comparison of Arab with non-Arab population. J Neurol Stroke 2016;4:00156 CrossRef

12. Beyrouti R, Adams ME, Benjamin L, et al. Characteristics of ischaemic stroke associated with COVID-19. J Neurol Neurosurg Psychiatry 2020;91:889-91 CrossRef Medline

13. Aggarwal G, Lippi G, Michael Henry B. Cerebrovascular disease is associated with an increased disease severity in patients with coronavirus disease 2019 (COVID-19): a pooled analysis of published literature. Int J Stroke 2020;15:385-89 CrossRef Medline

14. Avula A, Nalleballe K, Narula N, et al. COVID 19 presenting as stroke. Brain Behav Immun 2020;87:115-19 CrossRef Medline

15. Lodigiani C, Iapichino G, Carenzo L, et al; Humanitas COVID-19 Task Force. Venous and arterial thromboembolic complications in COVID-19 patients admitted to an academic hospital in Milan, Italy. Thromb Res 2020;191:9-14 CrossRef Medline

16. Chen $\mathrm{G}, \mathrm{Wu} \mathrm{D}$, Guo W, et al. Clinical and immunological features of severe and moderate coronavirus disease 2019. J Clin Invest 2020;130:2620-29 CrossRef Medline

17. Tang N, Li D, Wang X, et al. Abnormal coagulation parameters are associated with poor prognosis in patients with novel coronavirus pneumonia. J Thromb Haemost 2020;18:844-47 CrossRef Medline

18. Yaghi S, Ishida K, Torres J, et al. SARS2-CoV-2 and stroke in a New York healthcare system. Stroke 2020;51:2002-11 CrossRef Medline

19. Sweid A, Hammoud B, Weinberg JH, et al. Thrombotic neurovascular disease in COVID-19 patients. Neurosurgery 2020 June 4. [Epub ahead of print] CrossRef Medline

20. Kerleroux B, Fabacher T, Bricout N, et al; SFNR, the ETIS registry, and the JENI-Research Collaborative. Mechanical thrombectomy for acute ischemic stroke amid the COVID-19 outbreak: decreased activity, and increased care delays. Stroke 2020;51:2012-17 CrossRef Medline 